\title{
Microwave Interstellar Medium Emission in the Green Bank Galactic Plane Survey: Evidence for Spinning Dust
}

\section{Citation}

Finkbeiner, Douglas P., Glen I. Langston, and Anthony H. Minter. 2004. “Microwave Interstellar Medium Emission in the Green Bank Galactic Plane Survey: Evidence for Spinning Dust." The Astrophysical Journal 617 (1) (December 10): 350-359. doi:10.1086/425165.

\section{Published Version}

doi:10.1086/425165

\section{Permanent link}

http://nrs.harvard.edu/urn-3:HUL.InstRepos:33462889

\section{Terms of Use}

This article was downloaded from Harvard University's DASH repository, and is made available under the terms and conditions applicable to Other Posted Material, as set forth at http:// nrs.harvard.edu/urn-3:HUL.InstRepos:dash.current.terms-of-use\#LAA

\section{Share Your Story}

The Harvard community has made this article openly available.

Please share how this access benefits you. Submit a story.

\section{Accessibility}




\title{
MICROWAVE INTERSTELLAR MEDIUM EMISSION IN THE GREEN BANK GALACTIC PLANE SURVEY: EVIDENCE FOR SPINNING DUST
}

\author{
Douglas P. Finkbeiner ${ }^{1}$ \\ Department of Astrophysics, Princeton University, Peyton Hall, Princeton, NJ 08544 \\ AND \\ Glen I. Langston and Anthony H. Minter \\ National Radio Astronomy Observatory, P.O. Box 2, Green Bank, WV 24944 \\ Received 2003 September 12; accepted 2004 August 12
}

\begin{abstract}
We observe significant dust-correlated emission outside of $\mathrm{H}$ II regions in the Green Bank Galactic Plane Survey $\left(-4^{\circ}<b<4^{\circ}\right)$ at 8.35 and $14.35 \mathrm{GHz}$. The rising spectral slope rules out synchrotron and free-free emission as majority constituents at $14 \mathrm{GHz}$, and the amplitude is at least 500 times higher than expected thermal dust emission. When combined with the Rhodes $(2.326 \mathrm{GHz})$ and Wilkinson Microwave Anisotropy Probe (23-94 GHz) data, it is possible to fit dust-correlated emission at $2.3-94 \mathrm{GHz}$ with only soft synchrotron, free-free, thermal dust, and an additional dust-correlated component similar to Draine \& Lazarian spinning dust. The rising component generally dominates free-free and synchrotron for $\nu \gtrsim 14 \mathrm{GHz}$ and is overwhelmed by thermal dust at $\nu \gtrsim 60 \mathrm{GHz}$. The current data fulfill most of the criteria laid out by Finkbeiner and coworkers for detection of spinning dust.

Subject headings: diffuse radiation — dust, extinction — ISM: clouds — radiation mechanisms: thermal — radio continuum: ISM

Online material: color figure
\end{abstract}

\section{INTRODUCTION}

The statistical detection of anomalous dust-correlated emission, far brighter than expected thermal dust emission at $\sim 10$ $30 \mathrm{GHz}$, has been well established (COBE, Kogut et al. 1996; Saskatoon, de Oliveira-Costa et al. 1997; Owens Valley Radio Observatory [OVRO], Leitch et al. 1997; $19 \mathrm{GHz}$ survey, de Oliveira-Costa et al. 1998; Tenerife, de Oliveira-Costa et al. 1999). There has also been a convincing detection of this component in the dark cloud LDN 1622 (Lynds 1962) and detection of a rising spectrum in the diffuse $\mathrm{H}$ II region LPH 201.663+ 1.643 (Lockman et al. 1996) using the Green Bank 140 foot $(43 \mathrm{~m})$ telescope, but the interpretation as spinning dust is tentative (Finkbeiner et al. 2002). In most cases, free-free emission from ionized gas is ruled out as the source, either by a significantly rising spectrum or by morphological comparison with $\mathrm{H} \alpha$ maps. This anomalous dust-correlated emission has been called the "mystery component" or "Foreground X" (de Oliveira-Costa et al. 2002) to avoid premature interpretation of the signal. One possible explanation for this emission is electric dipole emission from rapidly $\left(\sim 1.5 \times 10^{10} \mathrm{~s}^{-1}\right)$ rotating small dust grains, known as "spinning dust" (Draine \& Lazarian 1998, hereafter DL98). Another possibility is magnetic dipole emission due to thermal fluctuations in the magnetization of magnetic grains (Draine \& Lazarian 1999). Henceforth, we follow de Oliveira-Costa and refer to "Foreground X," keeping these two mechanisms in mind as the most likely. Either mechanism might be the dominant source of dust-correlated microwave emission in the diffuse interstellar medium (ISM) at $\sim 15-60 \mathrm{GHz}$, overwhelmed by free-free and synchrotron emission at lower frequencies and nonmagnetic thermal dust emission at higher frequencies.
Even though Foreground $\mathrm{X}$ could be ubiquitous, severe limitations in previous data sets have prevented a convincing detection over a large solid angle. Surveys such as the $19.2 \mathrm{GHz}$ survey (Cottingham 1987; Boughn et al. 1992) and the Tenerife survey (Gutiérrez et al. 2000) have such large beams $\left(3^{\circ}\right.$ and $5^{\circ}$ FWHM, respectively) that any diffuse ISM emission in the plane is confused with $\mathrm{H}$ II regions, and, even when it can be detected by spectral slope (as in the Tenerife data at 10 and $15 \mathrm{GHz}$ ), there are relatively few independent pixels in the data showing rising emission. Other experiments, such as the COBE Differential Microwave Radiometer (DMR; $7^{\circ} \mathrm{FWHM}$ ), observed at higher frequencies at which the spinning-dust spectrum is expected to roll off and cannot be separated from free-free by spectral slope or spatial resolution. This has made it extremely difficult to argue for an unambiguous detection.

Recently, the Wilkinson Microwave Anisotropy Probe (WMAP; Bennett et al. 2003a) has produced high-sensitivity full-sky maps at $23,33,41,61$, and $94 \mathrm{GHz}$ from its first year of data. ${ }^{2}$ The main purpose of the mission is to determine cosmological parameters from cosmic microwave background (CMB) anisotropy, and this purpose has already been largely fulfilled (Hinshaw et al. 2003a; Spergel et al. 2003). In addition, the WMAP data provide full-sky maps of Milky Way emission in a frequency domain rarely explored before. To interpret this new foreground information, the WMAP team performed a maximum entropy method (MEM) analysis as well as more conventional template fitting and found that Foreground $\mathrm{X}$ is not necessary to fit the WMAP data; instead, they relied on a hard synchrotron component ( $T_{b} \sim \nu^{\beta}$ with $\beta \sim-2.5$, typical of supernova remnants) that is strongly correlated with dust (Bennett et al. $2003 \mathrm{~b}$ ). The spectrum of this hard synchrotron component (for $23 \mathrm{GHz}<\nu<94 \mathrm{GHz}$ ) is inconsistent with the DL98 cold

\footnotetext{
${ }^{1}$ Hubble Fellow, Henry Norris Russell Fellow.
}

${ }^{2}$ Available at http://lambda.gsfc.nasa.gov/product/map. 
neutral medium (CNM) model chosen for comparison but does not disagree strongly with a warm neutral medium (WNM) model scaled slightly in amplitude. Indeed, Finkbeiner (2004) demonstrates in a companion paper that the WMAP data off the plane $^{3}$ can be fitted by the following four components: (1) soft synchrotron, as traced by $408 \mathrm{MHz}$ (Haslam et al. 1982); (2) free-free, as traced by $\mathrm{H} \alpha$ emission in three recent surveys (VTSS, Dennison et al. 1998; SHASSA, Gaustad et al. 2001; and WHAM, Haffner et al. 2003) as presented by Finkbeiner (2003) plus an enhancement from hot gas within $30^{\circ}$ of the Galactic center; (3) Rayleigh-Jeans thermal (vibrational) dust emission as predicted by Finkbeiner et al. (1999, hereafter FDS99); and (4) a Foreground X template constructed from the FDS99 dust map times dust temperature squared. The spectral shape of each of the four components is not free to vary with position on the sky, as it is in the WMAP MEM analysis, yet the fit is superb. The spectrum derived for Foreground $\mathrm{X}$ can be explained by a superposition of the DL98 spinning-dust models, but it could also be explained as hard synchrotron highly correlated with dust.

Lagache (2003) has correlated the WMAP data with H I maps and found evidence for microwave emission in excess of that expected for synchrotron, free-free, and thermal dust. Banday et al. (2003) also address this excess in a new analysis of the $C O B E$ data and compare it to the WMAP findings. Recent measurements of the Helix planetary nebula at $26-36 \mathrm{GHz}$ with the Cosmic Background Imager also show a factor of 3 excess over the flux expected in the absence of Foreground $\mathrm{X}$ (Casassus et al. 2004). Finally, de Oliveira-Costa et al. (2004) cross-correlate the WMAP MEM synchrotron template (Bennett et al. 2003b) with Tenerife data at 10 and $15 \mathrm{GHz}$, finding that the correlation spectrum departs from the expected synchrotron shape by a factor of $\sim 10$ at $10 \mathrm{GHz}$. Unfortunately, all of these investigations have some shortcomings: none of the $W M A P$-based analyses have the power to rule out hard synchrotron as the Foreground $X$ component, because at least some spinning-dust models are degenerate with hard synchrotron over the frequencies observed $(\nu>23 \mathrm{GHz})$, leaving the interpretation of $W M A P$ ambiguous. A rising spectrum observed at lower frequencies, seen by de Oliveira-Costa et al. (2004), is stronger evidence, but the Tenerife data have a $5^{\circ}$ FWHM resolution. Fortunately, higher resolution data at appropriate frequencies do exist.

The Green Bank Galactic Plane Survey (Langston et al. 2000 ) at 8.35 and $14.35 \mathrm{GHz}$ provides key evidence in favor of Foreground $\mathrm{X}$, even though it was designed to detect transient point sources in the Galactic plane $\left(|b|<5^{\circ}\right)$, not to examine the diffuse ISM. The scan pattern is simpler than that desired for a diffuse ISM experiment, and in the original analysis the data stream was median filtered on a short timescale to reveal the point sources. However, the survey has the requisite resolution, sensitivity, and frequency coverage to make a highly significant detection of Foreground X. After careful reprocessing of the raw survey data, we have been able to produce images of this anomalous emission on a large angular scale and demonstrate that it is consistent with DL98 spinning dust, ruling out hard synchrotron as a majority component at $14 \mathrm{GHz}$ and $|b|<2^{\circ}$.

In this paper, we compare Foreground $\mathrm{X}$ to spinning dust because of theoretical bias (we know the small grains are there, and they have to spin and radiate at some level), keeping in

\footnotetext{
${ }^{3}$ Where $\mathrm{H} \alpha$ extinction is less than 2 mag, according to the SFD98 dust map.
}

mind that alternative interpretations, such as magnetic dipole emission, are possible.

In $\S 2$ we briefly describe the survey data. Section 3 describes the processing steps taken to produce maps of each "segment" of sky. Results based only on Green Bank data, using the full resolution (11.2) available, are presented in $\S 4$. Additional data from Rhodes and WMAP (smoothed to $1^{\circ}$ FWHM) are analyzed in $\S 5$, and conclusions are stated in $\S 6$.

\section{THE DATA}

The Green Bank Earth Station (GBES) is a $13.7 \mathrm{~m}$ dish equipped to communicate with satellites via two receivers centered at 8.35 (the $\mathrm{X}$ band) and 14.35 (the $\mathrm{Ku}$ band) GHz. These receivers view a common beam center on the sky (to within $1 / 8$ beam) through a dichroic element, allowing simultaneous observations at two frequencies. This simultaneity is advantageous for both satellite communication and transient source surveys but is also convenient for our purposes. The telescope resolution for point sources is $9 .^{\prime} 7 \pm 0 !^{\prime} 1$ at $8.35 \mathrm{GHz}$ and $6.6 \pm 0.2$ at $14.35 \mathrm{GHz}$. After convolving the data samples onto a grid, the effective resolution is 11.2 at $8.35 \mathrm{GHz}$ and 8.'0 at $14.35 \mathrm{GHz}$. Further details are given in Langston et al. (2000).

The scan strategy is to raster the telescope through $-5^{\circ}<$ $b<5^{\circ}$ along a line of (nearly) constant Galactic longitude, sampling total power (both circular polarizations) in a $500 \mathrm{MHz}$ bandwidth 9 times per second (every 2.'4) along the scan. Successive scans are spaced $5^{\prime}$ apart, Nyquist-sampling the sky at the $\mathrm{X}$ band but not at the $\mathrm{Ku}$ band. Data are taken in "segments" of 180 scans, covering a patch of $10^{\circ} \times 15^{\circ}$ on the sky. The segments are centered on integer multiples of $15^{\circ}$ Galactic longitude.

In order to find transient sources, all segments centered on $0^{\circ}<l<270^{\circ}$ were observed many times and compared. There are four complete surveys (GPA, GPB, GPC, and GPD), each of which nominally consists of four epochs a few days apart. Sometimes a given segment is observed several times within one epoch to compensate for bad weather. For the current project we use only GPA, which provides up to five observations of each segment, although in practice we choose to use the best three.

\section{PROCESSING STEPS}

\subsection{Segment Maps}

The processing begins with the gpa-*raw.fit files on $\mathrm{G}$. Langston's Web site. ${ }^{4}$ These files contain the time-ordered data, i.e., a set of sky coordinates $(l, b)$ and measurements in the $\mathrm{X}$ and $\mathrm{Ku}$ bands, as well as uncertainties. If the two bands had the same beam on the sky, it would be possible to compare them directly, but because the X-band beam is significantly larger than the Ku-band beam, it is necessary to make a map and smooth the $\mathrm{Ku}$-band beam before comparison.

Each set of 180 scans (covering $10^{\circ} \times 15^{\circ}$ ) constitutes a segment. Because the scans are not exactly on great circles, the data are mapped to an intermediate scan \#-b map, the $(n, b)$ grid, to facilitate Fourier destriping. The instrumental beam is very well sampled in the scan direction (samples every 2.'4), so linear interpolation in $b$ is sufficient to map to the $(n, b)$ grid. The image is significantly padded in the $b$-direction and Fourier-transformed, revealing significant power near the $k_{n}$ axis outside the band limit, representing stripes in the scan

\footnotetext{
${ }^{4}$ See http://www.gb.nrao.edu/ glangsto/gpa.
} 


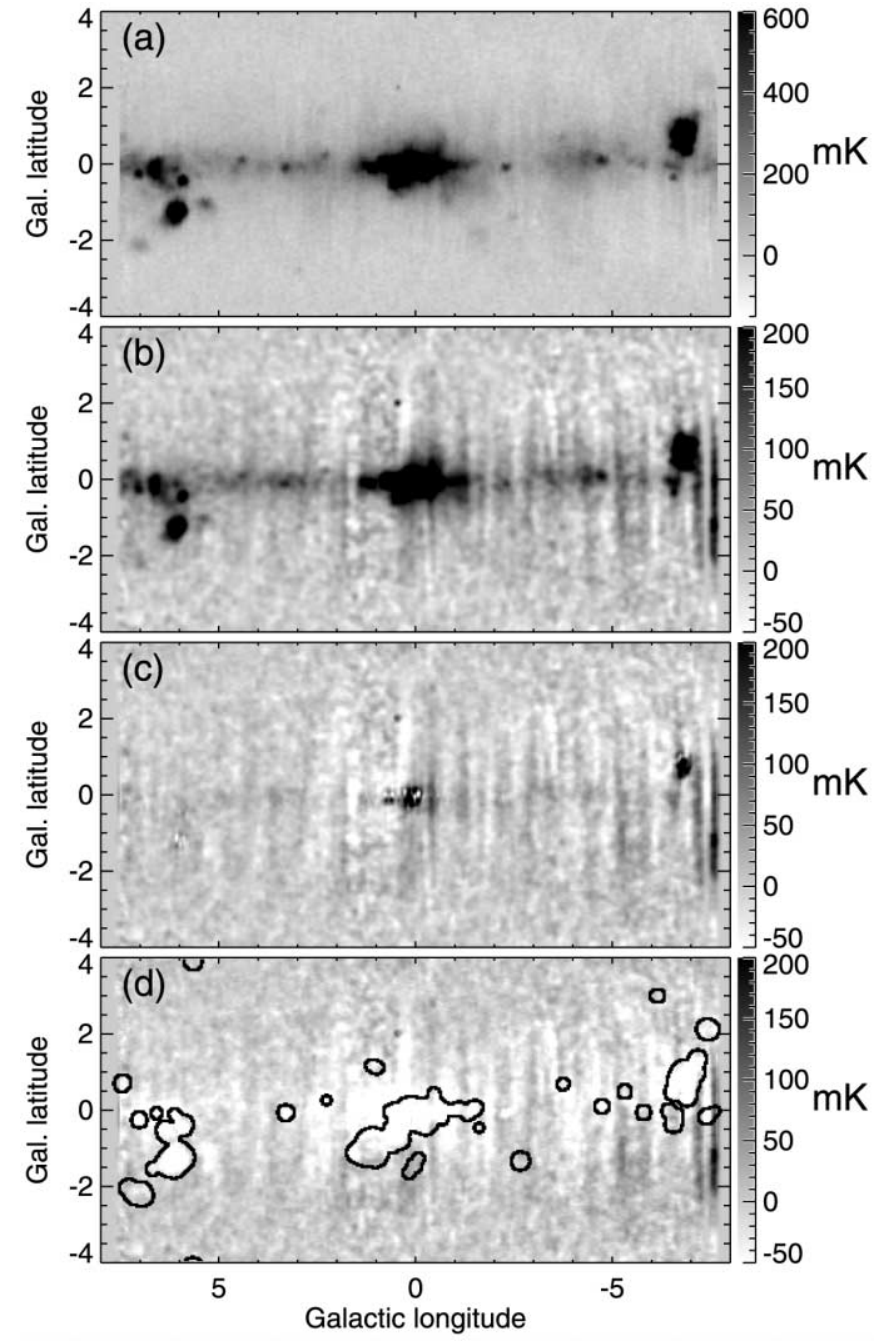

FIG. 1.-GPA segment centered on $l=0^{\circ}:(a)$ X-band $(8.35 \mathrm{GHz})$ map; $(b)$ $\mathrm{Ku}$-band $(14.35 \mathrm{GHz})$ map, smoothed to X-band beam; $(c)$ free-free subtracted Ku-band map, i.e., $T_{\mathrm{Ku}}-(14.35 / 8.35)^{-2.1} T_{\mathrm{X}} ;(d) T_{\mathrm{Ku}}-(14.35 / 8.35)^{-1.2} T_{\mathrm{X}}$ map with outline of $\mathrm{H}_{\text {II }}$ mask (see $\S 3.3$ ) superposed. The $\mathrm{H}_{\mathrm{II}}$ regions in $(d)$ are oversubtracted, but the diffuse emission is canceled. This segment exhibits more striping artifacts than the others.

direction. This power is subtracted, and the inverse Fourier transform produces a somewhat cleaner $(n, b)$ map.

After destriping, the data are interpolated along rows of constant $b$ from $(n, b)$ to $(l, b)$, a Cartesian grid with 2!'4 pixels. Again, a simple linear interpolation is used, because the X-band map is sampled well in this direction, and the Ku-band map, although not quite as well sampled, is noisy enough that linear interpolation does not compromise the data, especially considering the subsequent smoothing.

Segments centered on $l=0,15,30$, and 45 provide the strongest signal for the current work. We shall hereafter refer to these and other segments by their central longitude; all segments center on Galactic latitude 0 . Segment -10 is observed at low elevation and suffers from troublesome artifacts because of significant ground pickup coming through the sidelobes of the antenna. This raises the system temperature, resulting in "curved" features in the $(l, b)$ maps that are at a constant elevation above the horizon. These artifacts are understood and are not a problem for other parts of the sky. Segment 60 has a weak dust signal and poor weather. Segment 75 is dominated by free-free from a beautiful $\mathrm{H}$ II region in Cygnus but shows little diffuse ISM, and the remaining segments in the outer

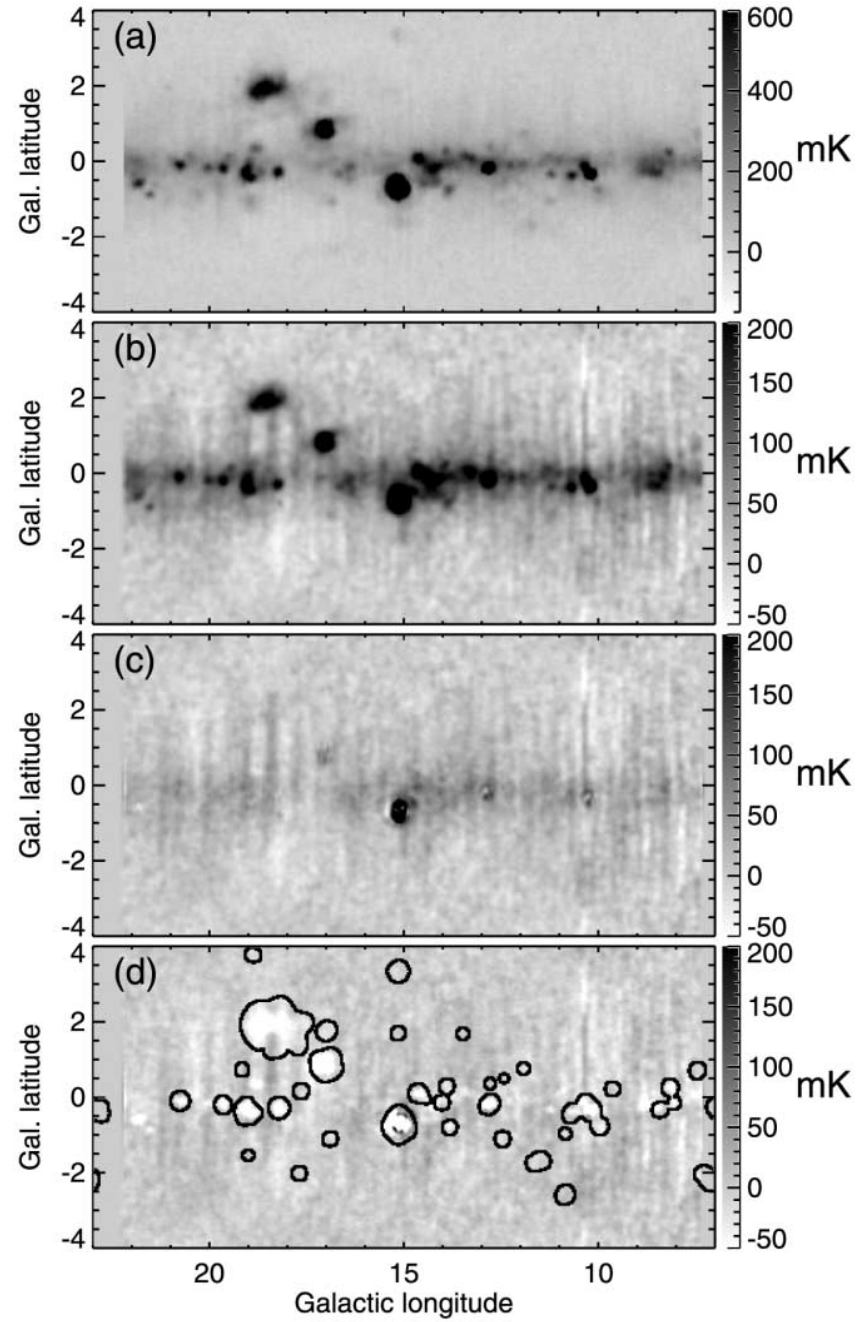

FIG. 2.- Same as Fig. 1, but for GPA segment centered on $l=15^{\circ}$. The subtraction is good in $(c)$, considering that the region around NGC 6618 at $(l, b)=15^{\circ} 1,-0.7$ is 10 times brighter than the region around NGC 6611 at $(l, b)=17^{\circ} .0,0^{\circ} .9$.

Galaxy show no significant ISM emission. Segment 0 is dominated by free-free but is included for completeness. Therefore, the results in this paper are derived exclusively from segments 15,30 , and 45 .

\subsection{Sky Subtraction}

The total antenna temperature (including system and sky contributions) for these observations is $68 \mathrm{~K}$ at the $\mathrm{X}$ band and $83 \mathrm{~K}$ at the $\mathrm{Ku}$ band (sum of both polarizations). The sky contribution to each sample $i$ is $T_{\mathrm{sky}, i}=T_{0}+T_{\text {sky }} \sec z_{i}$, where $z_{i}$ is the zenith angle of each sample $i$ and $T_{0}$ and $T_{\text {sky }}$ are constants fitted for each segment. This model is adequate in most cases, but in cases in which $T_{0}$ or $T_{\text {sky }}$ varies during the observation of one segment, a roughly quadratic error can be introduced into the maps. In addition, because the $\mathrm{X}$ and $\mathrm{Ku}$ bands are observed at the same time, the resulting artifacts will be highly correlated. Concerns about this prompted us to rereduce the data with a more "brute force" approach of fitting a quadratic function of $b$ (with $3 \sigma$ outlier rejection) to each scan off the plane $\left(|b|>2^{\circ}\right)$ and subtracting the fit. The resulting maps for segments $0,15,30$, and 45 are shown in Figures $1 a-$ $4 a$ (X band) and $1 b-4 b$ (Ku band). Note that the result of such fitting is to suppress any extended emission at $|b|>2^{\circ}$, but 


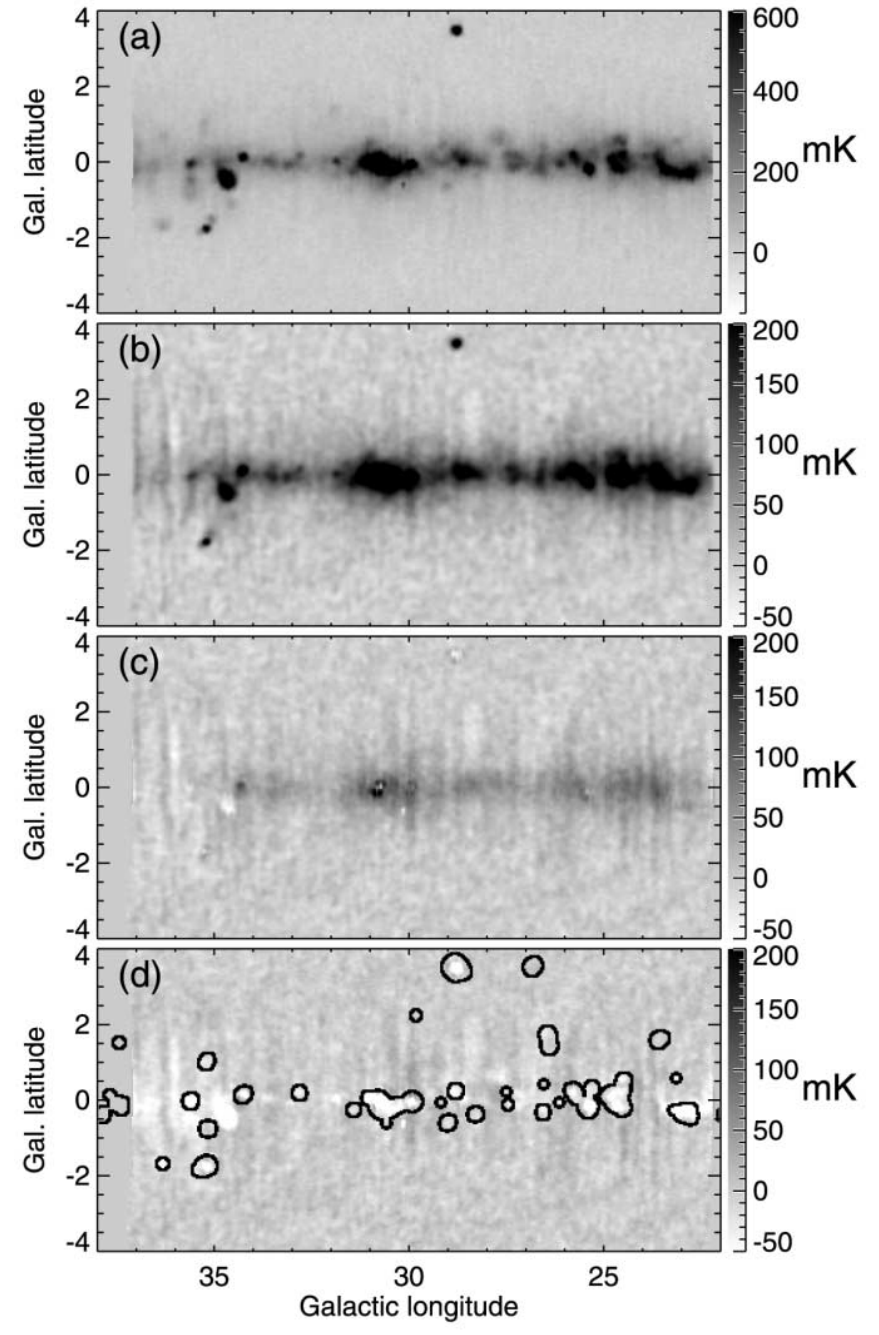

FIG. 3.- Same as Fig. 1, but for GPA segment centered on $l=30^{\circ}$. Oversubtracted regions in this map are dominated by synchrotron emission, such as the supernova remnant $3 \mathrm{C} 392$ at $(l, b)=34^{\circ} .75,-0.5$. The W40 complex is visible at $(l, b)=28^{\circ} .8,3^{\circ} .5$ and is used as a calibration check in $\S 5.5$.

such emission is negligible compared to emission in the plane anyway. Bright point sources such as W40 (segment 30) are preserved by outlier rejection in the baseline fit. Results in this study use this quadratic sky subtraction because it is less susceptible to systematic errors, but the results do not depend significantly on this choice.

\subsection{H II Region Mask}

The total emission in the Galactic plane is dominated by a few bright $\mathrm{H}$ II regions and supernova remnants. It is desirable to mask these out using independent data, such as the IRAS $60 / 100 \mu \mathrm{m}$ flux ratio (Beichman et al. 1988). This ratio is approximately $0.2-0.3$ in the diffuse ISM and exceeds $\sim 0.4$ in $\mathrm{H}$ II regions. A cut of 0.4 is taken and grown by 2 pixels in all directions. This mask is shown in outline in Figure $1 d$. Results are not very sensitive to this choice.

\section{RISING SPECTRUM FROM 8 TO $14 \mathrm{GHz}$}

The Green Bank data exhibit a rising intensity from 8 to $14 \mathrm{GHz}$ that cannot be explained by free-free, synchrotron, or thermal (vibrational) dust emission. Data for segments 0, 15, 30, and 45 are shown in Figures 1-4, respectively. In each case, the $\mathrm{X}$ - and $\mathrm{Ku}$-band images are shown, as well as two superpositions of the $\mathrm{X}$ - and Ku-band data: one that cancels free-free
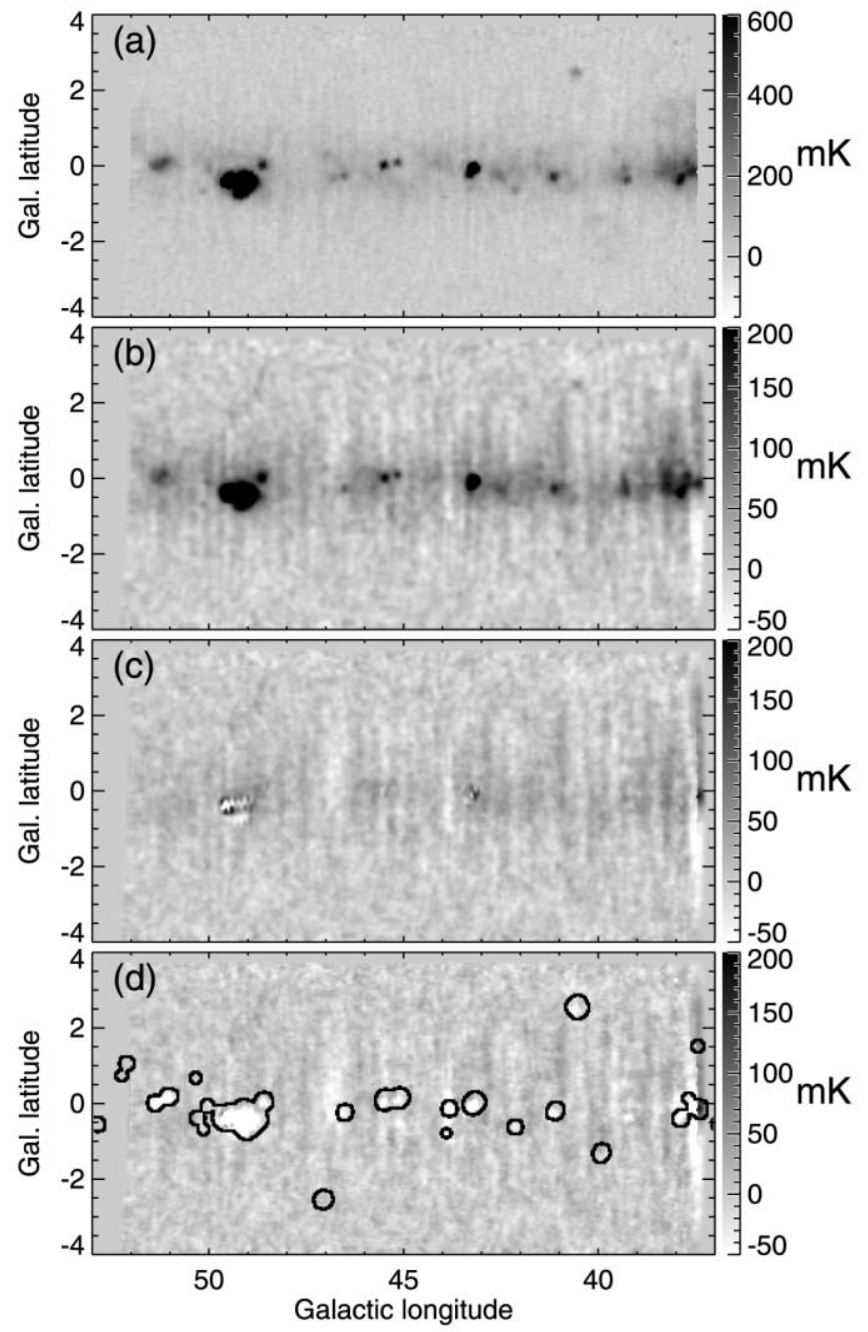

FIG. 4.- Same as Fig. 1, but for GPA segment centered on $l=45^{\circ}$.

$\left[T_{\mathrm{Ku}}-(14.35 / 8.35)^{-2.1} T_{\mathrm{X}}\right]$ and another that cancels the ISM in the Galactic plane outside of the IRAS-based H II mask. The relative calibration between the $\mathrm{X}$ and $\mathrm{Ku}$ bands is evidently good to better than $\sim 10 \%$ or the $\mathrm{H}$ iा regions would not be canceled by the first superposition so nicely. Note that segment 0 (Fig. 1) is dominated by free-free emission and difficult to interpret, but it is included for completeness.

A simple test reveals that the $\mathrm{X}$ - and $\mathrm{Ku}$-band data are consistent with spinning-dust models and not with free-free or synchrotron emission. We plot the free-free subtracted $\left[T_{\mathrm{Ku}}-\right.$ $\left.(14.35 / 8.35)^{-2.1} T_{\mathrm{X}}\right]$ superposition of the two bands versus the dust column (expressed as $100 \mu \mathrm{m}$ optical depth, $\tau_{100 \mu \mathrm{m}}=$ $266 \times E(B-V)$, from Schlegel et al. [1998, hereafter SFD98]) in Figure 5 and find a strong positive correlation for segments 15,30 , and 45 . Of course, a substantial amount of spinning dust would be canceled in this superposition, but an indirect comparison to the five DL98 models is possible by computing the same superposition for each model. The Draine \& Lazarian models are expressed as emissivity per $\mathrm{H}$ column density, so we convert $N(\mathrm{H})$ to $100 \mu \mathrm{m}$ optical depth using $2.13 \times 10^{24} \mathrm{H}$ $\mathrm{cm}^{-2}=$ unit $\tau_{100 \mu \mathrm{m}}$ to obtain a slope for each model. ${ }^{5}$ One

\footnotetext{
${ }^{5}$ This factor assumes a mean dust temperature of $18.175 \mathrm{~K}$ obtained by SFD98 and that a column density of $8 \times 10^{21} \mathrm{H} \mathrm{cm}^{-2}$ corresponds to $1 \mathrm{mag}$ $E(B-V)$, as derived by SFD 98 by comparing $21 \mathrm{~cm} \mathrm{H}$ I emission with dust FIR emission. This latter factor differs from the value of $5.8 \times 10^{21} \mathrm{H} \mathrm{cm}^{-2}$ reported by Bohlin et al. (1978).
} 

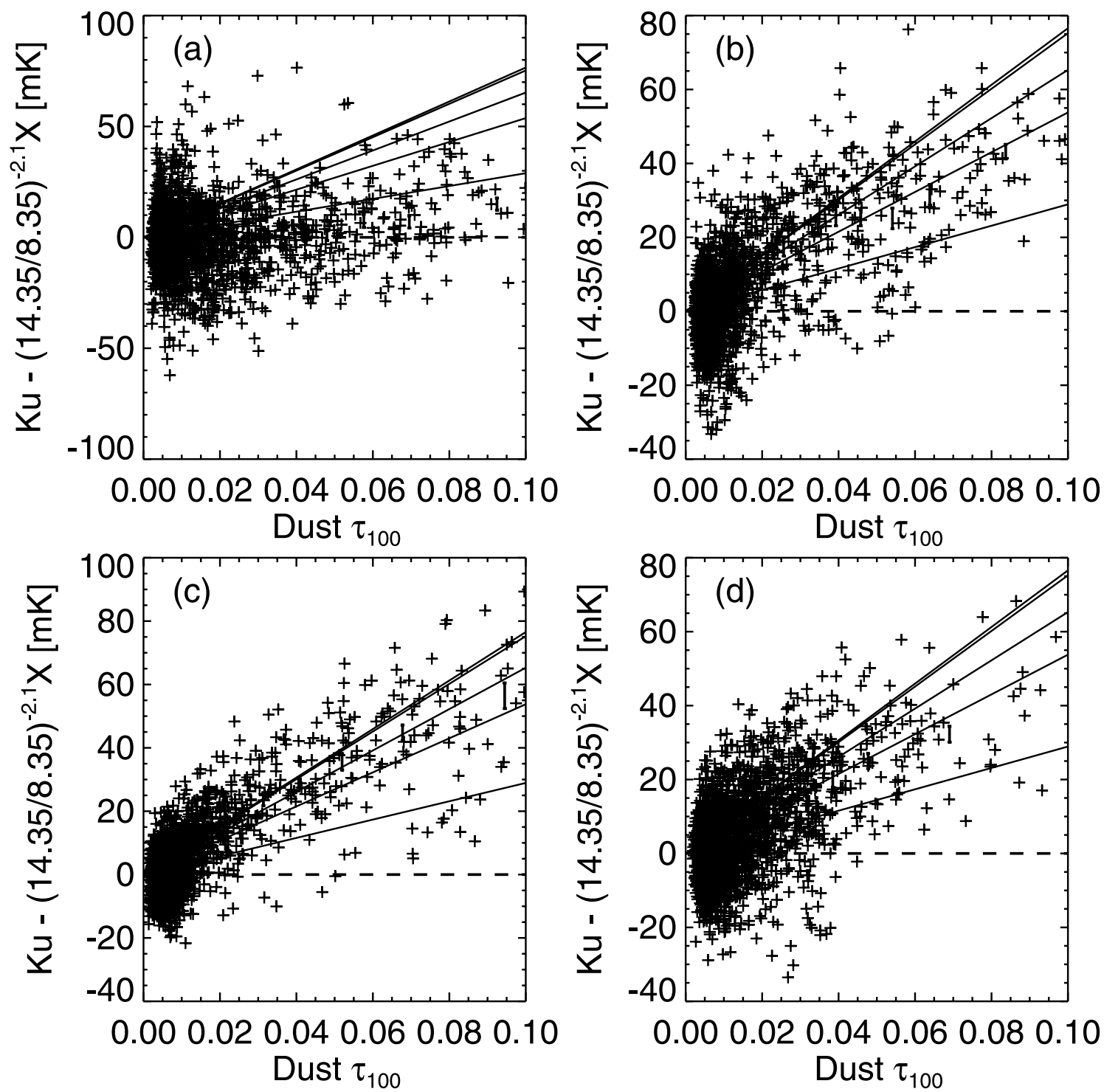

FIG. 5. - Free-free subtracted Ku-band antenna temperature vs. dust optical depth from SFD 98 for segments centered at $($ a $) 0^{\circ}$, (b) $15^{\circ}$, (c) $30^{\circ}$, and $(d) 45^{\circ}$ Galactic longitude. Each symbol represents a $12^{\prime} \times 12^{\prime}$ patch of sky (independent beams). Pixels dominated by free-free would fall on the dashed horizontal line and synchrotron would fall below the line in the absence of noise. The solid lines represent five DL98 spinning-dust models for (top to bottom) warm ionized medium (WIM), WNM, CNM, molecular clouds, and dark clouds. Error bars show $1 \sigma$ uncertainty of the mean values in bins containing 1/50 of the data points. These error bars do not include an overall calibration uncertainty of $10 \%$. Segments 15, 30, and 45 are consistent with spinning-dust models and inconsistent with free-free or synchrotron emission.

symbol is plotted for each $12^{\prime} \times 12^{\prime}$ independent region in each of the four segments; the vast majority fall among the five spinning-dust model lines. A region dominated by free-free would fall on the horizontal line; any emission with a slope steeper than $\beta=-2.1$ (such as hard synchrotron) would fall below the line. A few such regions appear, such as supernova remnant 3C 392 in segment 30, but in general most pixels outside the $\mathrm{H}$ II mask appear to be consistent with the spinningdust interpretation of Foreground $\mathrm{X}$ and inconsistent with freefree or synchrotron emission alone.

\section{GREEN BANK AND WMAP}

The previous section provided support for the spinning-dust hypothesis by demonstrating a rising spectrum in the Green Bank data from 8 to $14 \mathrm{GHz}$. By combining those data with the Rhodes survey (2.326 GHz; see Jonas et al. 1998) and WMAP (23-94 GHz), we can produce a spectrum of dust-correlated emission from 2.3 to $94 \mathrm{GHz}$.

\subsection{Choice of Dust Template}

By cross-correlating the data in each band against a dust template (such as SFD98) and measuring the correlation slope, we can obtain the desired information without being confused by sometimes poorly determined zero points of the various data sets. As a template we could use SFD98 dust (as in the previous section) or dust times some power of dust temperature (as in Finkbeiner 2004), but for the present analysis we prefer the WMAP $94 \mathrm{GHz}$ map. ${ }^{6}$ This map is dominated by thermal dust emission on the Rayleigh-Jeans tail, where temperature dependence is weak, so it is a good tracer of total dust-column density. This way the errors in the SFD98 dust-temperature correction at low Galactic latitude do not propagate into the analysis. All three templates have been used in the following

\footnotetext{
${ }^{6}$ The effective central frequency for the WMAP W band is $93.1 \mathrm{GHz}$ for free-free and $94.3 \mathrm{GHz}$ for dust. Because thermal dust is the dominant Galactic emission in the $\mathrm{W}$ band, we refer to it as the " $94 \mathrm{GHz}$ " band throughout.
} 

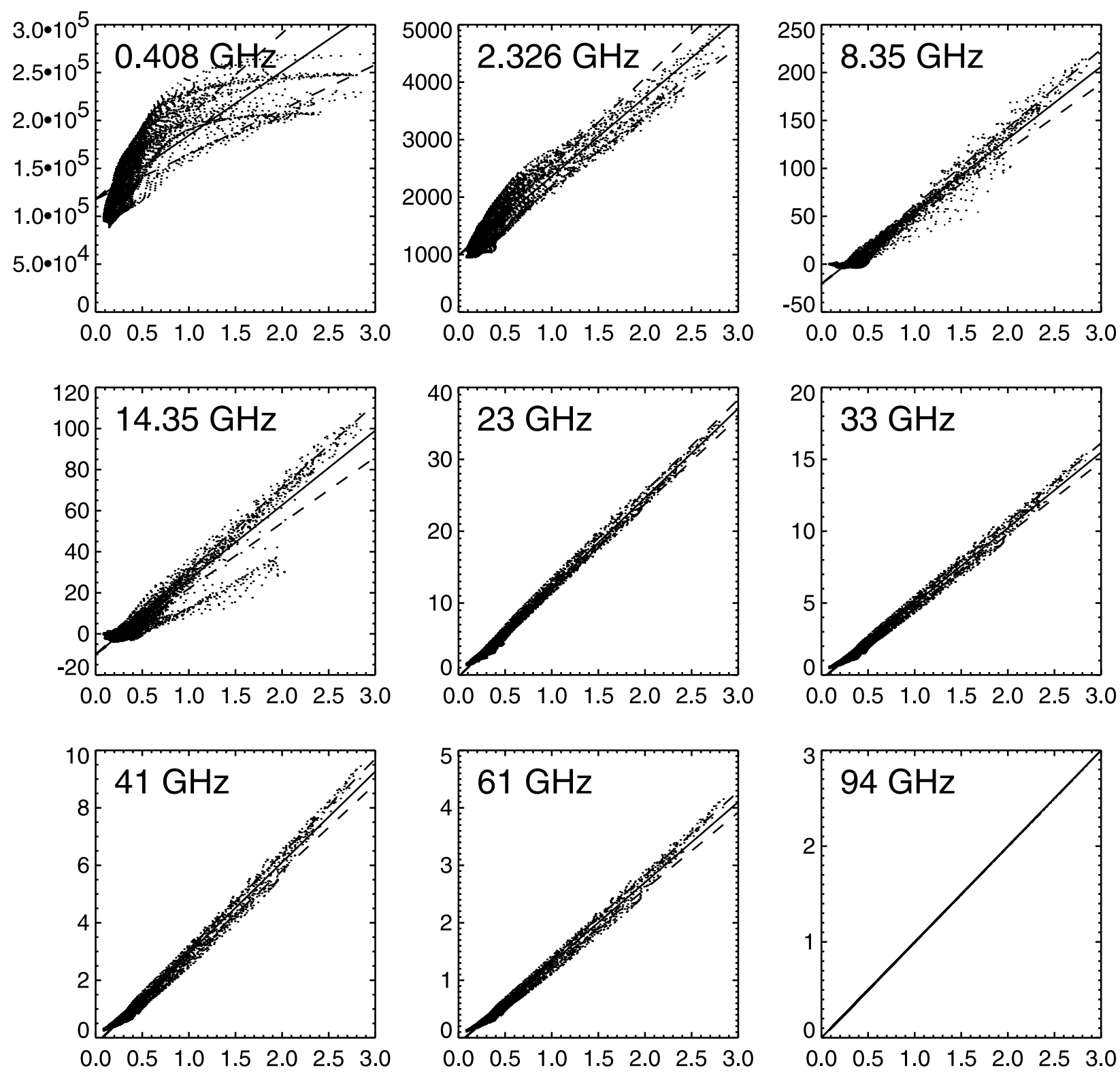

FIG. 6. - Scatter plots for segment 30. At each of nine frequencies, antenna temperatures (mK) are plotted vs. the WMAP $94 \mathrm{GHz}$ channel, used as a tracer of dust. CMB anisotropy is removed from the five $W M A P$ bands before plotting. When this plot is made using, e.g., SFD98 $100 \mu$ m optical depth as a dust tracer, the relative slopes are the same, but the scatter is greater. WMAP $94 \mathrm{GHz}$ is dominated by Rayleigh-Jeans dust emission and is therefore a good proxy for total dust-column density. Scatter plots like these were made for all four segments, and the correlation slopes are plotted vs. frequency in Fig. 7. We discard the Haslam $408 \mathrm{MHz}$ result because the scatter is so nonlinear.

analysis with nearly identical results, but the $94 \mathrm{GHz}$ map produces tighter scatter and small error bars.

\subsection{Correlation Slopes}

Scatter plots for each of nine channels $(0.408,2.326,8.35$, $14.35,23,33,41,61$, and $94 \mathrm{GHz}$ ) versus $94 \mathrm{GHz}$ are shown for segment 30 in Figure 6 along with best-fit linear regression lines. For this analysis, all maps are smoothed to $1^{\circ}$ FWHM, and the $\mathrm{H}$ II region mask is appropriately enlarged. Dashed lines indicate the $1 \sigma$ width of the distribution of slopes, not the uncertainty in the mean, which is much smaller.

These slopes are then converted to $j_{\nu} \tau_{100 \mu \mathrm{m}}^{-1}$ for comparison with results obtained in the previous section. The DL98 models refer to $N(\mathrm{H})$ because they are physical models tied to element abundances relative to $\mathrm{H}$. In practice, the microwave emissivities are compared to the dust optical depth (without regard for whether the associated $\mathrm{H}$ is molecular, neutral atomic, or ionized), and the models are converted from $j_{\nu} / n_{\mathrm{H}}\left(\mathrm{Jy} \mathrm{cm}^{2} \mathrm{sr}^{-1} \mathrm{H}^{-1}\right)$ to $j_{\nu} \tau_{100 \mu \mathrm{m}}^{-1}\left(\mathrm{Jy} \mathrm{sr}^{-1}\right)$ with a canonical factor of $2.13 \times 10^{24} \mathrm{H} \mathrm{cm}^{-2}=$ unit $\tau_{100 \mu \mathrm{m}}$. In the case of the $94 \mathrm{GHz}$ template, a conversion factor of $34.6 \mathrm{mK}^{7}$ per unit $\tau_{100 \mu \mathrm{m}}$ is applied, corresponding to $0.13 \mathrm{mK}$ per mag $E(B-V)$. This conversion factor can be fitted empirically from the SFD 98 maps and WMAP $94 \mathrm{GHz}$ data or derived from a comparison of the Far Infrared Absolute Spectrophotometer (FIRAS)-based $94 \mathrm{GHz}$ prediction of FDS99 and the SFD98 $\tau_{100 \mu \mathrm{m}}$ map for median dust temperature. Correlation slopes are fitted for the four segments, with segments 15,30 , and 45 showing a pronounced rise from 8 to $14 \mathrm{GHz}$ and requiring some sort of anomalous emission (Fig. 7).

\footnotetext{
${ }^{7}$ Antenna temperature, not thermodynamic $\Delta T$.
} 

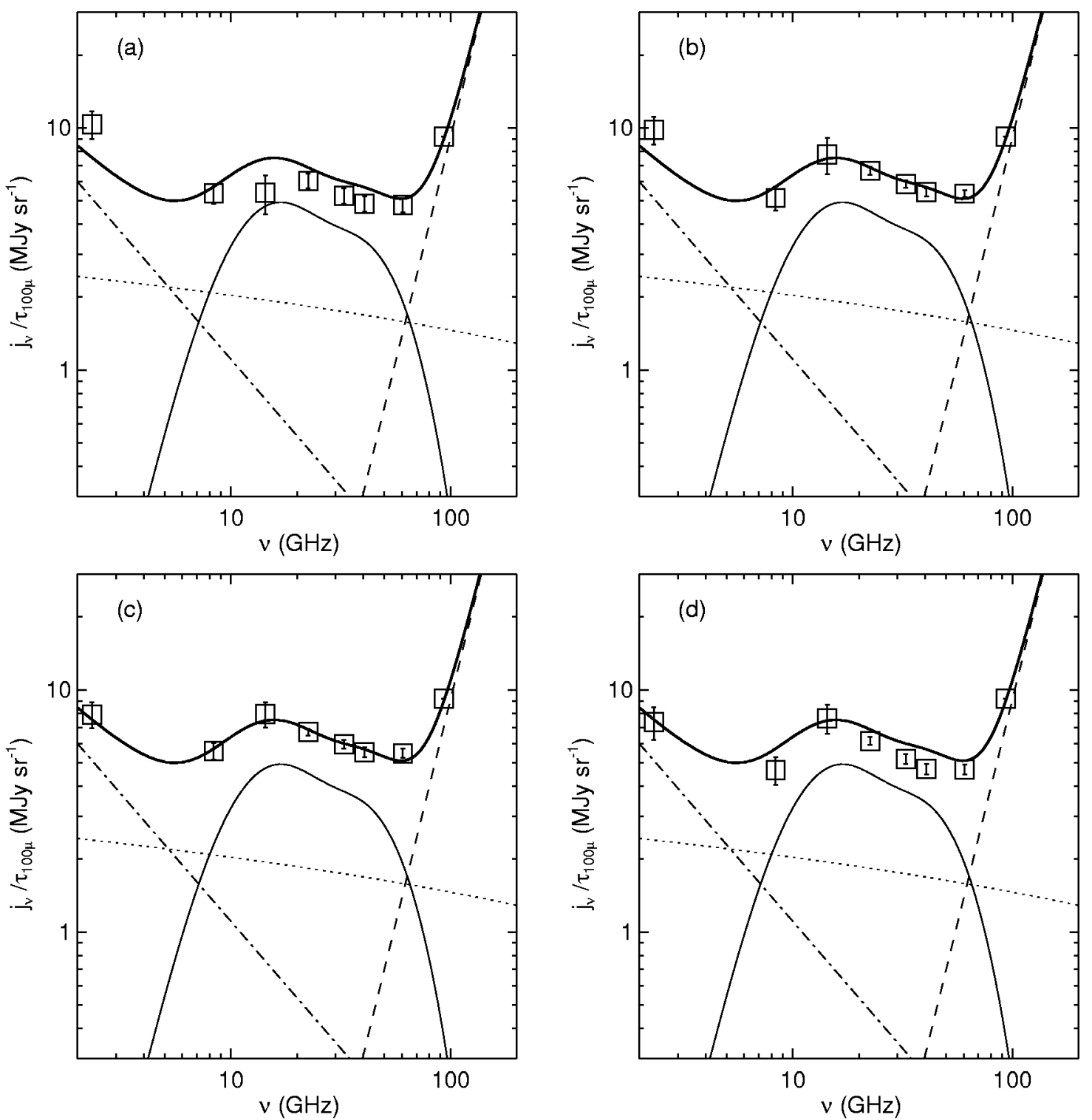

FIG. 7.-Correlation slope spectra for segments $(a) 0,(b) 15,(c) 30$, and $(d) 45$. The rise from 8 to $14 \mathrm{GHz}$ cannot be explained with any combination of free-free, synchrotron, and thermal dust. The $94 \mathrm{GHz}$ map is used as a tracer of dust-column density and is placed on this plot using a conversion factor calculated in $\S 5.2$. Also shown are free-free (dotted line), synchrotron with index $\alpha=-1.05$ (dash-dotted line), thermal dust with FDS99 calibration (dashed line), and a mock spinning-dust model described in $\S 5.2$ (thin solid curve), as well as the sum of these four components (thick solid curve). The same curves are overplotted in each panel to guide the eye and are not a formal fit to the data.

As a toy model, a superposition of 0.18 times the DL98 molecular cloud model plus 0.5 times the DL98 WNM spectrum is used, where the electric dipole moment is increased by a factor of 2 for the WNM curve. The WNM term is an extrapolation based on the DL98 WNM model for two values of the electric dipole moment and is not a rigorous calculation. This parameterization should not be taken seriously as a physical interpretation of the spectrum; it merely shows that a superposition of reasonable models provides a good fit to the data. Further study will be required to fit these curves in detail with a physical spinning-dust model.

\subsection{Magnetic Dust}

Another emission mechanism that could produce Foreground X is magnetic dust (Draine \& Lazarian 1999). This mechanism has nothing to do with rapid rotation of magnetic dipoles; rather, it results from the thermal fluctuations in the grain magnetization, yielding an additional thermal emissivity mechanism. The published Draine \& Lazarian (1999) models have a slow rolloff above the peak intensity, and this rolloff results in high theoretical $61 \mathrm{GHz}$ correlation slopes relative to the WMAP data. That is, for the measured $61 \mathrm{GHz}$ to be so low, a sharp rolloff in the underlying dust spectrum with increasing frequency is required. However, it may be possible to tune the dust magnetic susceptibility function to produce a steep rolloff (B. T. Draine 2002, private communication). We have not explored this parameter space but simply note that while the published magnetic models appear to be ruled out by the data, a variant of such models may not be ruled out as a majority contribution. Further theoretical work is required before the dominant dust-correlated emission mechanism can be unambiguously identified. What is certain, however, is that it is not hard synchrotron emission alone. 
TABLE 1

Correlation Slope Results

\begin{tabular}{|c|c|c|c|c|c|}
\hline $\begin{array}{c}\nu \\
(\mathrm{GHz})\end{array}$ & $\begin{array}{c}\text { Seg 0 } \\
\left(j_{\nu} / \tau_{100 \mu \mathrm{m}}\right)\end{array}$ & $\begin{array}{c}\text { Seg } 15 \\
\left(j_{\nu} / \tau_{100 \mu \mathrm{m}}\right)\end{array}$ & $\begin{array}{c}\text { Seg } 30 \\
\left(j_{\nu} / \tau_{100 \mu \mathrm{m}}\right)\end{array}$ & $\begin{array}{c}\text { Seg } 45 \\
\left(j_{\nu} / \tau_{100 \mu \mathrm{m}}\right)\end{array}$ & $\begin{array}{c}\mathrm{W} 40 \\
\left(j_{\nu} / \tau_{100 \mu \mathrm{m}}\right)\end{array}$ \\
\hline 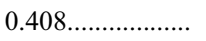 & $18.30 \pm 5.60$ & $18.75 \pm 5.71$ & $11.80 \pm 3.52$ & $13.37 \pm 4.23$ & $11.33 \pm 5.00$ \\
\hline $2.326 \ldots \ldots \ldots \ldots \ldots$ & $10.33 \pm 1.36$ & $9.81 \pm 1.31$ & $7.90 \pm 0.96$ & $7.36 \pm 1.14$ & $17.68 \pm 1.16$ \\
\hline 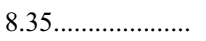 & $5.37 \pm 0.51$ & $5.13 \pm 0.57$ & $5.60 \pm 0.44$ & $4.67 \pm 0.61$ & $12.56 \pm 1.18$ \\
\hline 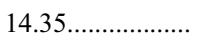 & $5.38 \pm 0.99$ & $7.75 \pm 1.34$ & $7.95 \pm 0.95$ & $7.62 \pm 1.05$ & $12.61 \pm 1.14$ \\
\hline $22.5 \ldots \ldots \ldots \ldots \ldots \ldots$ & $6.05 \pm 0.43$ & $6.65 \pm 0.25$ & $6.69 \pm 0.23$ & $6.15 \pm 0.24$ & $12.59 \pm 0.41$ \\
\hline $32.7 \ldots \ldots \ldots \ldots \ldots \ldots$ & $5.24 \pm 0.39$ & $5.86 \pm 0.21$ & $5.97 \pm 0.25$ & $5.19 \pm 0.24$ & $12.87 \pm 0.39$ \\
\hline 40.6......................... & $4.86 \pm 0.36$ & $5.42 \pm 0.20$ & $5.53 \pm 0.26$ & $4.70 \pm 0.23$ & $12.72 \pm 0.43$ \\
\hline 60.7 ........................ & $4.81 \pm 0.33$ & $5.35 \pm 0.18$ & $5.47 \pm 0.23$ & $4.69 \pm 0.21$ & $12.37 \pm 0.36$ \\
\hline 93.1.................... & $9.20 \pm 0.00$ & $9.20 \pm 0.00$ & $9.20 \pm 0.00$ & $9.20 \pm 0.00$ & $16.57 \pm 0.00$ \\
\hline
\end{tabular}

Note.-Correlation slopes (emission per dust $100 \mu \mathrm{m}$ optical depth, $\left.\tau_{100 \mu \mathrm{m}}\right)$ for Haslam $(0.408 \mathrm{GHz})$, Rhodes $(2.326 \mathrm{GHz})$, Green Bank $(8.35$ and $14.35 \mathrm{GHz})$, and WMAP $(22.5-93.1 \mathrm{GHz})$ in units of $\mathrm{MJy} \mathrm{s}^{-1}$. To convert to emissivity per $\mathrm{H}$ atom $\left(\mathrm{Jy} \mathrm{cm}^{2} \mathrm{sr}^{-1} \mathrm{H}^{-1}\right)$, divide by $2.13 \times 10^{18}$. Error values represent the $1 \sigma$ width of the distribution of slopes as seen in Fig. 6, not the (much smaller) uncertainty of the mean. The WMAP bands are rather broad; the listed WMAP frequencies are effective central frequencies for emission with a free-free spectrum given by Page et al. (2003). The highest frequency WMAP band, dominated by thermal dust emission, is used as the "dust template" in this paper and has no measured error. The W40 spectrum is substantially contaminated by free-free emission at $94 \mathrm{GHz}$, resulting in a higher normalization (see $\S 5.5$ for details). Conversion from WMAP thermal dust to $\tau_{100 \mu \mathrm{m}}$ is described in $\S 5.2$.

\subsection{Limits on Hard Synchrotron}

The WMAP team (Bennett et al. 2003b) claims that hard synchrotron $(\beta=-2.5)$ emission from dusty star-forming regions is the main contributor to dust-correlated emission at $23 \mathrm{GHz}$. They arrived at this conclusion without the benefit of any diffuse ISM measurements of the Milky Way between 2.3 and $23 \mathrm{GHz}$. Now that a rise in the dust spectrum has been observed in the 8 and $14 \mathrm{GHz}$ Green Bank data, it is evident that another emission mechanism is required. By assuming that Foreground $\mathrm{X}$ contributes nothing at $8 \mathrm{GHz}$ and rises sharply to $14 \mathrm{GHz}$ (and ignoring all other components, such as freefree), one can make the conservative statement that hard synchrotron is less than two-thirds of the $14 \mathrm{GHz}$ intensity and less than one-half at $23 \mathrm{GHz}$ (based on the data in Table 1). Inclusion of free-free or soft synchrotron pushes these limits down further. We therefore conclude that dust-correlated hard synchrotron provides less than one-half the dust-correlated intensity in the WMAP bands, and possibly much less.

\subsection{Calibration Check: W40}

The arguments in this paper depend most heavily on the spectral rise observed in the 8 and $14 \mathrm{GHz}$ Green Bank maps; thus, the calibration of these maps deserves close scrutiny. In order to verify the calibration of the Green Bank data relative to $W M A P$ (which has a calibration uncertainty of less than $1 \%$; Hinshaw et al. 2003b) we single out the $\mathrm{H}$ II region W40 at $(l, b)=28^{\circ} .8,3.5$ and repeat the above analysis. W40 is an association of at least six luminous stars adjacent to a molecular cloud (see Smith et al. 1985 for details) and is certainly an inhomogeneous environment. However, at the scale of comparison $\left(1^{\circ}\right)$, it is unresolved. WMAP $94 \mathrm{GHz}$ data are again used as a dust template, but all correlation slopes are multiplied by 1.8 (i.e., $94 \mathrm{GHz}$ emission per $\tau_{100 \mu \mathrm{m}}$ is high by that factor here, determined by a fit to the W40 spectrum) to account for the fact that only part of the $94 \mathrm{GHz}$ emission in this $\mathrm{H}$ II region is actually thermal dust. The W40 spectrum (Fig. 8) appears to be mostly free-free over a wide spectral range, with a small but significant enhancement above $33 \mathrm{GHz}$. The spinning-dust component included for comparison is the DL98 molecular cloud model times a factor of 0.15 . This is close to the factor of 0.2 suggested by DL98 to account for the expected depletion of small grains in molecular clouds. This plot demonstrates that (at least in segment 30) the Green Bank calibration (Langston et al. 2000) appears to be compatible with $W M A P$ at the $10 \%$ level or better. This indicates that the disagreement with the hard synchrotron hypothesis is robust and does not result from calibration error. The correlation slope results from Green Bank and WMAP (Table 1) as well as other experiments are compared to the DL98 models in Figure 9.

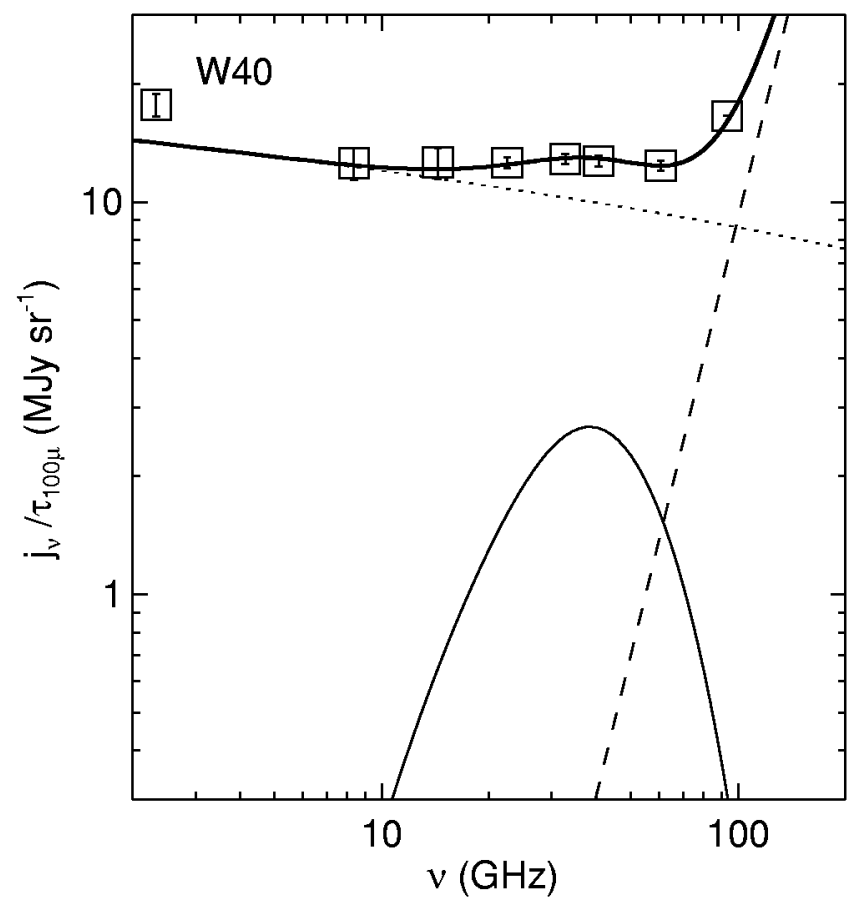

FIG. 8.-Correlation slope spectrum of W40. W40 is an $\mathrm{H}$ II region around six or more OB stars eating into a large molecular cloud. The dominance of free-free and distance from the Galactic plane $(3.5)$ allow this source to be used as a calibration check. The curve through the data points is a sum of freefree (dotted line), thermal dust (dashed line), and 0.15 times the DL98 model for molecular cloud spinning dust (thin solid line). This is close to the factor of 0.2 proposed by DL98 to account for the expected depletion of small grains in molecular clouds, but this plot should not be overinterpreted; the point is that the Green Bank 8.35 and $14.35 \mathrm{GHz}$ data and the WMAP data have a consistent calibration. 


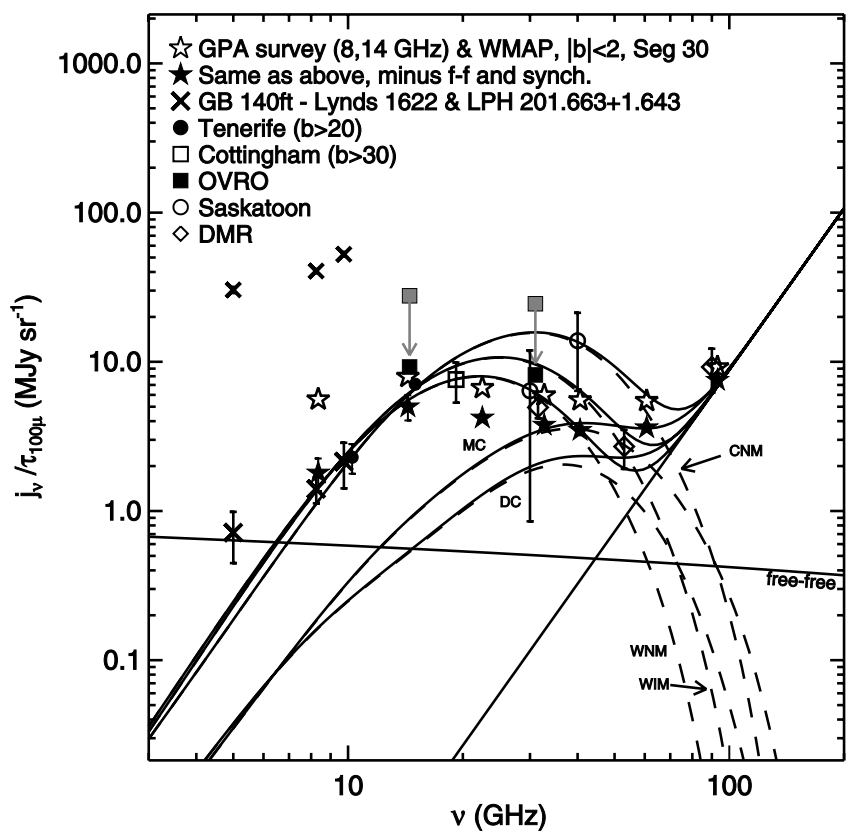

FIG. 9.-Model dust emissivity per $\tau_{100 \mu}$ for DC, MC, CNM, WNM, and WIM conditions (as in DL98, Fig. 9). Solid lines are total emissivity; dashed lines are rotational emission. Solid horizontal line is free-free for $\left\langle n_{e} n_{p}\right\rangle /\left\langle n_{\mathrm{H}}\right\rangle=$ $0.01 \mathrm{~cm}^{-3}$ averaged along the line of sight. Also shown are measurements from the COBE/DMR (open diamonds; FDS99), similar to Kogut et al. (1996); Saskatoon (open circles; de Oliveira-Costa et al. 1997); the Cottingham \& Boughn 19.2 GHz survey (open square; de Oliveira-Costa et al. 1998); OVRO data ( filled squares; Leitch et al. 1997); Tenerife data ( filled circles; de OliveiraCosta et al. 1999); GB 140 data for LPH 201.663+1.643 (upper crosses) and LDN 1622 (lower crosses; Finkbeiner et al. 2002); and Green Bank/WMAP data for segment 30 from this study with synchrotron and free-free included (open stars) and removed (filled stars), assuming free-free is $10^{-18} \mathrm{Jy} \mathrm{cm}^{2} \mathrm{sr}^{-1} \mathrm{H}^{-1}$ at 8.35 $\mathrm{GHz}$ and synchrotron is $2.4 \times 10^{-18} \mathrm{Jy} \mathrm{cm}^{2} \mathrm{sr}^{-1} \mathrm{H}^{-1}$ at $2.326 \mathrm{GHz}$ with $\alpha=-1.05$, as in Fig. 7. The OVRO points have been lowered by a factor of 3 relative to DL98 (Fig. 9), because the unusual dust temperature near the north celestial pole caused an underestimate of the $\mathrm{H}$ column density along those lines of sight. Given the large range of model curves, most measurements are consistent with some superposition of spinning dust, vibrational dust, and free-free emission. [See the electronic edition of the Journal for a color version of this figure.]

\subsection{Sidelobes}

Substantial contribution from ground pickup in beam sidelobes is always a potential concern with continuum measurements. At $14 \mathrm{GHz}$, the brightest regions on the sky are of the order of $1 \mathrm{~K}$, and the ground is $300 \mathrm{~K}$, so ground emission is far more important than reflection of the sky. Such ground emission does appear in the segment centered at $l=-10$, creating an obvious constant elevation pattern, so this segment has been discarded.

Far sidelobes on the sky are not a concern, because the sky baseline for each scan is established by fitting each scan at the ends, $|b|>2^{\circ}$, with a quadratic fit. The far (tens of degrees) sidelobes can only produce a very smooth background and are subtracted by this fit. The only potential contamination comes from the near sidelobes (a few degrees off-axis) picking up bright objects on the sky.

To verify that the near sidelobes are negligible, we scanned the unresolved source Cas A from eight approach angles, i.e., every $45^{\circ}$ (Figs. 10 and 11). Artifacts away from the main beam, such as diffraction spikes, are at the few percent level. There is no sign of the second Airy ring, which for a $2 \mathrm{~m}$ secondary mirror blocking the $13.7 \mathrm{~m}$ primary would theoretically have an amplitude of $2.4 \%$ of the peak and contain

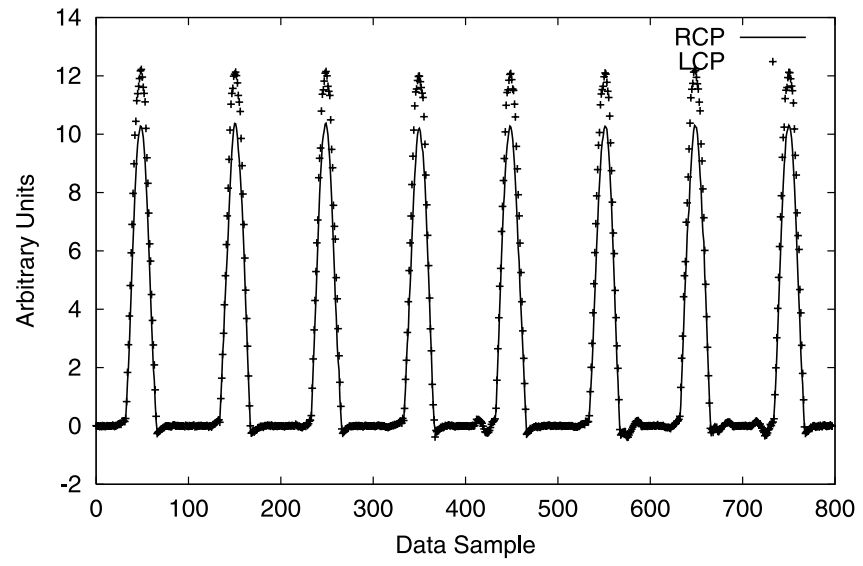

FIG. 10.-X-band beam map of Cas A scanning at eight different approach angles $\left(\right.$ every $45^{\circ}$ ). Samples are taken every $30^{\prime \prime}$. Power in each polarization is shown in arbitrary units. Hysteresis may be present at the $2 \%$ level, with other artifacts (diffraction spikes) in some scans at the 3\%-4\% level. No Airy ring structure is evident at less than $1 \%$ in amplitude, meaning less than $4 \%$ power in the first Airy ring.

$10 \%$ of the beam power. Where the scans are slightly off-center, diffraction spikes of 3\% amplitude can be seen in Figure 11 near peaks 5 and 6 , so the unseen sidelobes are probably less than $1 \%$. This happens because the illumination of the primary is not uniform but rather rolls off to near zero at the edge of the dish. This apodization suppresses the Airy rings, making the amplitude less than the theoretical $2.4 \%$. A $1 \%$ amplitude (which seems conservative) implies that $4 \%$ or less of the total power is scattered into the first Airy ring. Unfortunately, this telescope is no longer in service, so more complete beam maps are impossible to obtain. It is difficult to imagine sidelobe contamination affecting our correlation slopes by more than a few percent, compared with the factor of 2 discrepancy between the $\mathrm{X}$ - and Ku-band data and the Bennett et al. (2003b) synchrotron model.

\section{SUMMARY}

A previous analysis (Finkbeiner 2004) showed that WMAP data off the Galactic plane are consistent with spinning-dust

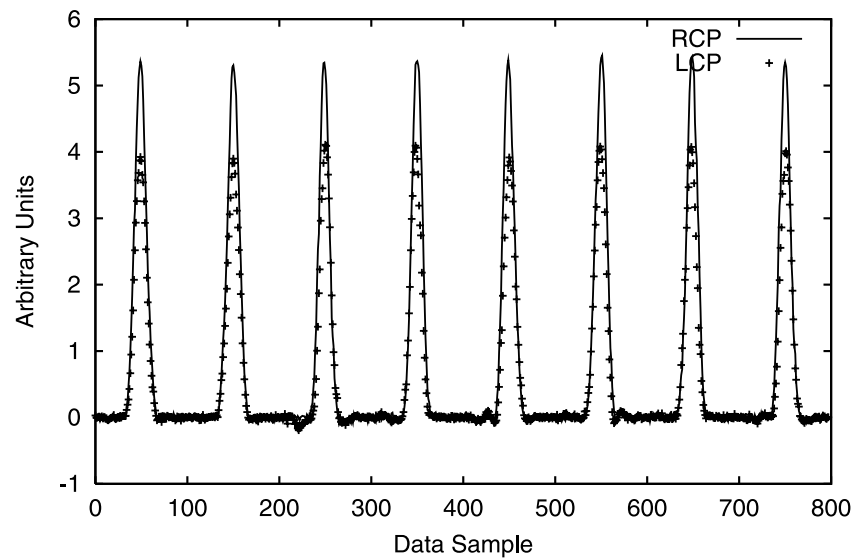

FIG. 11.-Ku-band beam map of Cas A scanning at eight different approach angles (every $45^{\circ}$ ). Samples are taken every $30^{\prime \prime}$. Power in each polarization is shown in arbitrary units. The hysteresis seen in the $\mathrm{X}$ band is absent, but diffraction spikes $( \pm 3 \%)$ appear next to peaks 5 and 6 because that scan was slightly off-center. A data glitch appears before peak 3 in one polarization. Sidelobes are estimated to be less than $1 \%$ in amplitude, meaning less than $4 \%$ power in the first Airy ring. 
emission similar to that proposed by DL98 but agreed with Bennett et al. (2003b) that a dust-correlated hard synchrotron model $(\beta \sim-2.5)$ could explain the emission as well. WMAP alone cannot differentiate between these two possibilities because the spectral rise predicted for most spinning-dust models occurs at lower frequencies $(\nu<23 \mathrm{GHz})$. Because there are no other data of comparable quality at high latitude in the required frequency range, this study makes use of the Green Bank Galactic Plane Survey $(8.35$ and $14.35 \mathrm{GHz})$ at low latitude $\left(-4^{\circ}<b<\right.$ $4^{\circ}$ ), finding a significant spectral rise and ruling out hard synchrotron as a majority contributor at $23 \mathrm{GHz}$. In light of this discovery, it is likely that Foreground X dominates hard synchrotron at higher latitudes as well.

Previous attempts to understand microwave foregrounds have missed important features in the data (e.g., by ignoring Foreground $\mathrm{X}$ altogether, ignoring effects of dust-temperature variation, or failing to identify the Galactic haze discovered by Finkbeiner 2004), and we may continue to oversimplify by assuming the same physical mechanism is responsible for all occurrences of Foreground X. In this early phase of the investigation, we have attempted to keep the analysis described herein (fitting correlation slopes as a function of frequency) as straightforward as possible, aiming to demonstrate the exis- tence of Foreground $\mathrm{X}$ and motivate further measurements. More sophisticated techniques may be used in the future to refine our understanding of Foreground(s) $\mathrm{X}$ and to derive parameters of physical models for the emission (as in DL98). For example, polarization measurements of clouds such as LDN 1622 at $3-10 \mathrm{GHz}$ may constrain spinning-dust models (see Lazarian \& Draine 2000). The present analysis simply argues strongly for the existence of spinning dust or another component with similar spectral behavior.

We are indebted to Bruce Draine and David Schlegel for encouragement and advice. Carl Heiles and Angelica de Oliveira-Costa provided helpful conversations. This research made use of the NASA Astrophysics Data System (ADS) and the IDL Astronomy User's Library at Goddard Space Flight Center (available at http://idlastro.gsfc.nasa.gov). D. P. F. is a Hubble Fellow supported by HST-HF-00129.01-A and NASA LTSA grant NAG5-12972. The Green Bank Earth Station telescope is located at Green Bank Observatory, operated by the National Radio Astronomy Observatory (NRAO), which is a facility of the National Science Foundation operated under cooperative agreement by Associated Universities, Inc.
Banday, A. J., Dickinson, C., Davies, R. D., Davis, R. J., \& Górski, K. M. 2003, MNRAS, 345, 897

Beichman, C. A., Neugebauer, G., Habing, H. J., Clegg, P. E., \& Chester, T. J., eds. 1988, IRAS Catalogs and Atlases, Explanatory Supplement (Washington: GPO)

Bennett, C. L., et al. 2003a, ApJ, 583, 1 2003b, ApJS, 148, 97

Bohlin, R. C., Savage, B. D., \& Drake, J. F. 1978, ApJ, 224, 132

Boughn, S. P., Cheng, E. S., Cottingham, D. A., \& Fixsen, D. J. 1992, ApJ, 391, L49

Casassus, S., et al. 2004, ApJ, 603, 599

Cottingham, D. A. 1987, Ph.D. thesis, Princeton Univ.

Dennison, B., Simonetti, J. H., \& Topasna, G. 1998, Publ. Astron. Soc. Australia, 15,147

de Oliveira-Costa, A., Kogut, A., Devlin, M. J., Netterfield, C. B., Page, L. A., \& Wollack, E. J. 1997, ApJ, 482, L17

de Oliveira-Costa, A., Tegmark, M., Page, L., \& Boughn, S. 1998, ApJ, 509, L9

de Oliveira-Costa, A., et al. 1999, ApJ, 527, L9

. 2002, ApJ, 567, 363

2004, ApJ, 606, L89

Draine, B. T., \& Lazarian, A. 1998, ApJ, 508, 157 (DL98)

. 1999, ApJ, 512, 740

Finkbeiner, D. P. 2003, ApJS, 146, 407

. 2004, ApJ, 614, 186

Finkbeiner, D. P., Davis, M., \& Schlegel, D. J. 1999, ApJ, 524, 867 (FDS99)

\section{EFERENCES}

Finkbeiner, D. P., Schlegel, D. J., Frank, C., \& Heiles, C. 2002, ApJ, 566, 898 Gaustad, J. E., McCullough, P. R., Rosing, W., \& Van Buren, D. 2001, PASP, 113,1326

Gutiérrez, C. M., Rebolo, R., Watson, R. A., Davies, R. D., Jones, A. W., \& Lasenby, A. N. 2000, ApJ, 529, 47

Haffner, L. M., Reynolds, R. J., Tufte, S. L., Madsen, G. J., Jaehnig, K. P., \& Percival, J. W. 2003, ApJS, 149, 405

Haslam, C. G. T., Stoffel, H., Salter, C. J., \& Wilson, W. E. 1982, A\&AS, 47, 1 Hinshaw, G., et al. 2003a, ApJS, 148, 135

. 2003b, ApJS, 148, 63

Jonas, J. L., Baart, E. E., \& Nicolson, G. D. 1998, MNRAS, 297, 977

Kogut, A., et al. 1996, ApJ, 464, L5

Lagache, G. 2003, A\&A, 405, 813

Langston, G., Minter, A., D’Addario, L., Eberhardt, K., Koski, K., \& Zuber, J. 2000, AJ, 119, 2801

Lazarian, A., \& Draine, B. T. 2000, ApJ, 536, L15

Leitch, E. M., Readhead, A. C. S., Pearson, T. J., \& Myers, S. T. 1997, ApJ, 486, L23

Lockman, F. J., Pisano, D. J., \& Howard, G. J. 1996, ApJ, 472, 173

Lynds, B. T. 1962, ApJS, 7, 1

Page, L., et al. 2003, ApJS, 148, 39

Schlegel, D. J., Finkbeiner, D. P., \& Davis M. 1998, ApJ, 500, 525 (SFD98)

Smith, J., Bentley, A., Castelaz, M., Gehrz, R. D., Grasdalen, G. L., \& Hackwell, J. A. 1985, ApJ, 291, 571

Spergel, D. N., et al. 2003, ApJS, 148, 175 